\title{
Realization of Brain Fingerprinting Using P300
}

\author{
Umesh K. Vidhyapriya R, Balaji ${ }^{1}$ \\ \{umeshkuttikrishnan@yahoo.com, rvidhyapriya@gmail.com, balajipsg26@gmail.com¹ \\ Department of Biomedical Engineering, PSG College of Technology ${ }^{1}$
}

\begin{abstract}
EEG is a physiological signal observed in the brain as a summation of discrete electrical activity emanating from every cellular element constituting the brain. Several vital information are hidden inside an EEG signal, which can be unearthed according to our need provided by suitable mathematical models and processing. In this research work, a feature called as P300 has been extracted from the EEG waveform and proved its usage for fingerprinting the brain. Several applications can be coined with the developed algorithm, which stretching from forensic to exoskeleton command triggering. The EEG signals were taken from 120 healthy individual and tested with the standard testing protocol used in the medical industry. Wavelet transforms and neural network are implemented in the research work for efficient extraction of the P300. The accuracy obtained with the neural network is promising to be used for the applications under consideration.
\end{abstract}

Keywords: EEG, P300, Brain Fingerprint, MERMER, Daubechies wavelet.

\section{Introduction}

The human nervous system is a complex functional arrangement that performs various tasks needed for the survival. The brain is an integral part of the nervous system that executes and processes much of the functionalities. The bulk of the human brain is the cranium, which looks similar to other animals but three times larger in proportion to the body size. Controlling, processing, planning and reasoning are performed at various lobes of the brain. There is a significant difference in the dimensions of associated centers in human brain when comparing to other species.

When analyzing the encephalization, the proportion of the brain to body size is varied dramatically during the evolution of various species. The number of neuron that constitutes human brain ranges up to hundreds of billions. The $10 \%$ of the neurons forms the cortical pyramidal cells. The nervous interconnections are called as synapses. There are around 1000 trillion synaptic connections in the human brain. The final processing of all the sensory information is carried out at the brain. The brain is also responsible for regulating all of the vital functions like breathing, pumping of blood and state of mind. Deep thinking, memory and learning ability are termed as the significant property of human brain which differentiates from the other animals. Neocortex is responsible for such activities.

The nature have provide us with vide range of protection for the brain, which includes skull, cerebrospinal fluid and blood brain barrier. However, the possibility of the brain injury still exists with various actions of humans in his day to day activities. Poisoning, injury and physical damage can happen to brain at any time which all affects the functions of brain 
drastically. Apart from the external damages, there is a growing possibility of internal causes for brain damage such as neurodegenerative diseases such as Parkinson's disease, Alzheimer's, sclerosis etc. Now a days, certain state of mind conditions such as depression and schizophrenia are all associated with the functions of brain.

The electrical potentials emerging from the brain is termed as electroencephalogram (EEG). The EEG provides vital information related to the state of brain. The recording, filtering and the location of the electrodes for acquiring the EEG is the trickiest part in the electro encephalogram. The amplitude of such a signal will be of few hundreds of micro volts. Several frequency ranges are split in the EEG signals in order to characterize the waves for analysis purpose.

\subsection{Brain Fingerprinting}

The brain fingerprinting is a general term used to determine the information of interest is being stored in the brain and also the mechanism to retrieve it. Multiple techniques and mathematical manipulations might be needed to precisely map the brain for an intended purpose [5]. EEG sensors fitted at specific location will influence the output of the brain mapping. Memory and Encoding Related Multifaceted Electroencephalographic Response (MERMER), is a test employed with the EEG signals to detect the similarity of knowing by an individual. P300 is a specific wave pattern exhibited in the EEG wave after a stimulus [6]. The 300 is the term represents the milliseconds. The major advantage of analyzing the P300 is that the emotions of the individual will not get reflected in the wave strip. This make the P300 more promising feature to be used in brain mapping, especially in forensic applications [7].The stimulus used as a trigger in the recording can be of different forms, which includes sound pattern, light, vibrations, pain etc. The mapping function will finally categorize as known, unknown and no response [8].

\subsection{Literature Review}

Numerous researches had been published in various research journal beas on P300 and Brain mapping. Much of the research was focused on the extraction of P300 and also on its application to predict or identify specific feature of interest.

Mahnazarvaneh et al. in their research work developed a P300 based training tool [1]. They have employed neurofeedback in their research and proved that they acquired reduced distraction by non-target population. The research is directed towards the proof of concept and an investigative model.

Ali Haider et al. in his research work, the P300 is utilized as a specific pattern which act as a scale for the mechanism with which the stimulus is computed [2]. The claimed their technique is stable in the way it is performed. Several applications of the P300 had been demonstrated by them in their work.

In the research work published by Jianing Li et al., P300 hads been employed for specific attention of tactile information [3]. Their research is directed towards the somatosensory excitation. Their work claims that the finger can be used as a promising tool for brain computer interfacing. They also try to prove that the proposed technique is not interfere with the visual or auditory functions.

Lukasz smigielski et al., in their research work the P300 is directed towards the selfreferential process [4]. They focused the work for psilocybin cure. In their work, the analysis had been carried out for assessing the impact of treatment. A study on scalp and its terrain have been analyzed.

From the literature it is evident that the P300 wave can be used for efficient tool for selecting, predicting and processing the brain related activities [9]. With the knowledge, it is proposing the P300 for predicting the known and unknown information for an individual. 


\section{Materials And Methods}



Figure 1. Methodology

The major steps involved in the research work are EEG signal acquisition, filtering the signal, correlation and neural network implementation as shown in figure 1. The system finally predicts the person under study knows particular information or not.

\subsection{IDENTIFICATION AND VERIFICATIONOF P300}

From the literature it is evident that the P300 exist at the delta region of the EEG. The absolute filtering of Delta wave can be utilized for the extraction of P300 wave. In the research work the initial task employed is to filter the Delta wave. In order to provide maximum flexibility in design the EEG signal is acquired as a raw data and the delta has been filtered digitally. Evoked potential is carried out for 120 individual using the EEG machine.Theverification of the P300 in the signal is verified from the PSG hospitals, Coimbatore, Tamilnadu, India.The acquisition was carried out with 10-20 lead system and RMS EEG machine, which measures the evoked potentials. The acquisition of P300 wave is performed with auditory stimulus using auditory evoked potential and also acquired P300 signals with visual stimulus using visual evoked potential onreal time basis using RMS EEG machine.

\subsection{SIGNIFICANCE OF P300}

$>$ Prominent P300 signals can be visualized for Visual stimulus rather than with Auditory stimulus.

$>$ For identical stimulus the P300 responses are different.

$>$ Wavelet transform can make the P300 more visible to analyse.

$>$ As far as it includes for more number of test images, the P300 can be easily distinguishable.

2.3 SIGNAL ACQUISITION

ST 2355 manufactured by Scientech has been used to acquire the EEG signal. Three scalp electrode as shown in figure 2 and NI-DAQ have been employed to acquire the signal to PC. 
For a better pre-amplification, three operational amplifiers and instrumentation amplifiers have been used. The input impedancewere kept at $1000 \mathrm{M} \Omega$. The frequency response is selected between $1 \mathrm{H}_{\mathrm{Z}}$ to $10 \mathrm{KH}_{\mathrm{Z}}$. The low pass filter is an integral part of the circuitry. The gain of the main amplifier is selected from 400 to 1000 . In consideration with the user friendliness the scalp electrode has been chosen with the positive electrode at occipital lobe $\mathrm{Oz}$ and the reference electrode at the forehead. The negative terminal is at frontal/parietal lobe (Fz/Pz). The P300 is more prominent at the negative electrode.

\subsection{SIGNAL PROCESSING}

Apart from the analog implementation of filter, several digital manipulation of signal is essential for precise revelation of P300 from the raw EEG signal. The wavelet transform is used as a fundamental signal processing tool for filtering out the P300. For better distinguishing the signal from the EEG several other signal parameters have been calculated which includes mean, absolute value, variance, standard deviation, zero crossing, slope sine change, energy entropy and normalization of signal.

\subsection{NEURAL NETWORK}

Extreme learning machine (ELM) neural network architecture was chosen as the methods for prediction. Since it is based on random projection, the suitable output can be visualized from the above mentioned technique. More over the ELM is extremely fast no over fitting problem as far as the application was concerned. It is also have less computational complexity than several other techniques.

\section{Results And Discussion}

\subsection{Types of Stimulus}

\section{A. Type 1:}

The type of stimulus is based on the nature of the test being carried out. In this research work a series of known and unknown pictures are flashed on the front screen of the subject. The subject is allowed to see the information. The constraint with such a method is that, it response created cannot distinguish whether it is due to the flash of the light during the display of the picture or is it truly due to the viewing of picture.

\section{B. Type 2:}

A predefined slideshowhas been prepared with different pictures, with frequent known and unknown to the subject. The slides were prepared by an individual who have knowledge about the person under test. A time limit of one sec was allotted for each slide. During the slideshow, the subject was recorded with the EEG. By this manner the recording is enabled with the exact timings which correlate between the triggering modality and the response.

There has been a prominent difference in the accuracy for both type of stimulus type as mentioned in the table 1 . The accuracy is increased in the case with both the known and the unknown is mixed before displaying to the subjects.

\subsection{FEATURE VALUES COMPARISON}

The features and its value are depicted in table 2 and table 3 . The table shows significant variations in the type of stimulus. The total length of the signal is selected as 200 samples. The comparison is performed both with two known and unknown picture separately and also combined. The power 




Figure 2: Electrode placement

Table 1: Accuracy of output with different stimulus type

\begin{tabular}{|c|c|c|}
\hline [1] Sl. No. & [2] Type of stimulus & [3] Accuracy \\
\hline$[4] 1$ & $\begin{array}{l}\text { [5] Continuous known } \\
\text { pictures and continuous } \\
\text { unknown pictures }\end{array}$ & [6] $50 \%$ \\
\hline$[7] 2$ & $\begin{array}{l}\text { [8] Continuous unknown } \\
\text { pictures with rare known } \\
\text { pictures. }\end{array}$ & [9] $70 \%$ \\
\hline$[10] 3$ & $\begin{array}{l}\text { [11] Set of three pictures } \\
\text { (Known, unknown and } \\
\text { mix) }\end{array}$ & {$[12] 95 \%$} \\
\hline
\end{tabular}

Table 3: Correlation coefficients

\begin{tabular}{|c|c|c|}
\hline $\begin{array}{c}{[13] \text { KNOWN }} \\
\text { CORR. } \\
\text { COEF }\end{array}$ & $\begin{array}{c}\text { [14] UNKNOWN } \\
\text { CORR. COEF }\end{array}$ & {$[15]$ Result } \\
\hline$[16] 0.53315$ & {$[17] 0.827808$} & {$[18]$ Unknown } \\
\hline$[19] 0.702669$ & {$[20] 0.844031$} & {$[21]$ Unknown } \\
\hline$[22]-0.70654$ & {$[23] 0.051755$} & {$[24]$ Unknown } \\
\hline$[25]-0.52393$ & {$[26] 0.957802$} & {$[27]$ Unknown } \\
\hline$[28] 0.779876$ & {$[29]-0.71466$} & {$[30]$ Known } \\
\hline
\end{tabular}




\begin{tabular}{|c|c|c|}
\hline$[31]-0.68964$ & {$[32] 0.253775$} & {$[33]$ Unkown } \\
\hline$[34] 0.462417$ & {$[35]-0.53551$} & {$[36]$ Known } \\
\hline$[37] 0.816168$ & {$[38] 0.141285$} & {$[39]$ Known } \\
\hline$[40] 0.429946$ & {$[41]-0.61785$} & {$[42]$ Known } \\
\hline
\end{tabular}

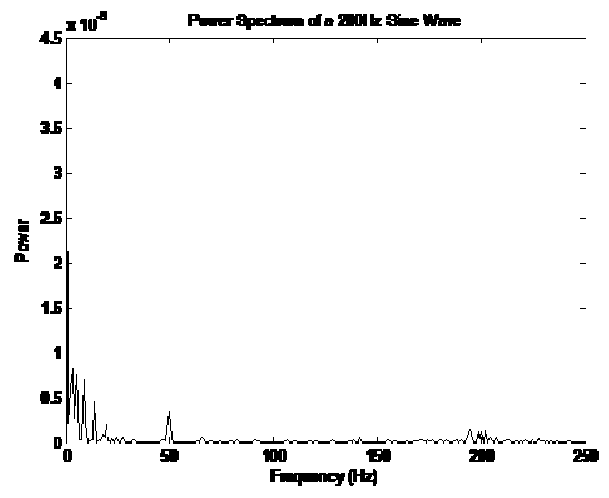

Figure 3: Power spectrum



Figure 4: Filtered EEG 


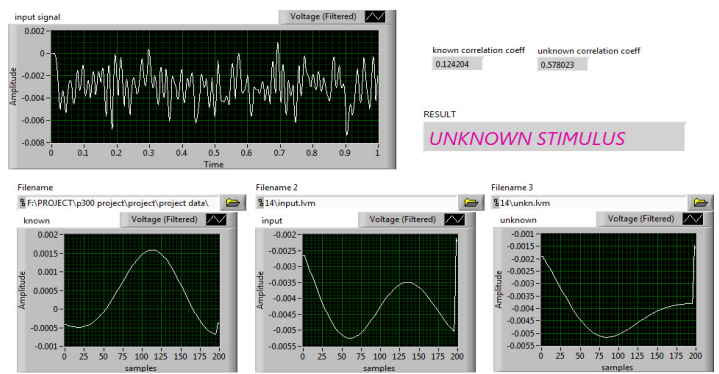

Figure 5: User interface

Spectrum for the signal is shown in figure 3.The P300 extracted at different stimulus type are distinguishable at every feature type. This is enabled with the wavelet transform performed at the initial decomposition of the signal. The Daubechies ( $\mathrm{Db} 4)$ is the suitable mother wavelet chosen for P300 extraction. A gradual change of the feature is observed for mean, variance, energy, entropy and normalized value. The values for energy were found to be high in known stimulusrather than in unknown.

\subsection{FILTERING THE EEG}

The faithfulness of the system to yield the best possible result is based on the filtering the EEG signal. Daubechies wavelet is identified to the transform that yield the best result for extracting. Figure 4 shows the effectiveness in filtering the EEG for different decomposition levels of Daubechies wavelet.

\subsection{FEATURES TO TRAIN THE NEURAL NETWORK}

Several features have been employed in this research work under the neural network. The major parameters are energy, $4^{\text {th }}$ Delta coefficient and signal averaging. The correlation coefficient is considered as the feature to perform the training of the neural network. The efficiency was appeared to be higher in this case.

\subsection{TRAINING AND TESTING PERFORMANCE}

The confusion matrix for the training and testing data set has been mentioned in table 4 and 5. The overall training efficiency is obtained as $98 \%$ and the testing accuracy is $100 \%$. For the binary operation the result encompasses on the ability of the feature to predict the probability of known and unknown element for an individual. Thus the brain mapping is faithfully realized using the proposed technique.

Table 4: Confusion matrix for training data set

\begin{tabular}{|l|l|l|}
\hline$[43]$ & {$[44] \mathrm{K} 1$} & {$[45] \mathrm{UK} 1$} \\
\hline$[46] \mathrm{K} 1$ & {$[47] 66$} & {$[48] 3$} \\
\hline$[49] \mathrm{UK} 1$ & {$[50] 0$} & {$[51] 93$} \\
\hline
\end{tabular}

Table 5: Confusion matrix for testing data set

\begin{tabular}{|l|l|l|}
\hline$[52]$ & {$[53] \mathrm{K} 1$} & {$[54] \mathrm{UK} 1$} \\
\hline
\end{tabular}




\begin{tabular}{|l|l|l|}
\hline$[55] \mathrm{K} 1$ & {$[56] 33$} & {$[57] 0$} \\
\hline$[58] \mathrm{UK} 1$ & {$[59] 0$} & {$[60] 22$} \\
\hline
\end{tabular}

\section{Conclusion And Future Work}

P300 component of EEG was successfully extracted from the raw EEG using the conventional recording system and digital filters. The wavelet has been designed to best fit for extracting the P300. A neural network was also developed and the output accuracy pertaining to its training and test data set have been plot. The system validated to be fit for brain mapping. In the future, much more data can be incorporated and validate to improve the efficiency. Different methods of stimulus can also be incorporated and test for extension of applications.

\section{Funding}

We thank the AICTE (All India Council for Technical Education, No. 8-38/RIFD/RPSNDF/Policy-1/2018-19) for the financial support provided through Research Promotion Scheme for this research work.

\section{References}

[1] MahnazArvaneh, Ian H Robertson, Tomas E ward, "A P300 Based Brain-Computer Interface for Improving Attention”, Frontiers in human neuroscience, 2019.

[2] Ali haider, Reza fazelRezai, "Application of P300 event related potential in brain computer interface", Event related potential and evoked potentials, 2017.

[3] Jianing Li, JiangboPu et al., " An online P300 Brain computer interface based on tactile selective attention of somatosensory electrical stimulation", Journal of medical and biological engineering, 2019.

[4] Lukasz smigielski, Michel kometer et al., "P300 mediated modulations in self-other processing under psychedelic psilocybin are related to connectedness and changed meaning: A window into the self-other overlap", Human Brain mapping, 2020.

[5] Turnip, Arjon, DwiEsti K et al., "Brain Mapping of Low and High Implusivity based P300 Signals", Journal of Physics, 2018.

[6] Ashford, J. W., Coburn, K. L., Rose, T. L., and Bayley, P. J."P300 Energy Loss in Aging and Alzheimer's Disease". J. Alzheimer's Dis., 2011

[7] Finke, A., Lenhardt, A., and Ritter, H., T'hemindgame: a p300-based brain-computer interface game." Neural Networking, 2009.

[8] Kelly, S. P., and O 'connell, R. G. "Behavioral/cognitive internal and external influences on the rate of sensory evidence accumulation in the human brain" J. Neuroscience, 2013.

[9] Kleih, S., Nijboer, F., Halder, S., and Kübler, A. "Motivation modulates the p300 amplitude during brain-computer interface use.", Clinical Neurophysiology, 2010.

[10] Dr.Gayathri Monicka, Apurva,Nishant and Piorebello "Design and Development of a Smart Electric Trike with detachable handle for Differently Abled" Elsevier material today . https://doi.org/10.1016/j.matpr.2020.06.261.

[11] Amutha devi, Gayathri Monicka.J, "Emerging bio-medical applications and open research challenges in cognitive internet of things (CIOT)" International Journal of Pharmacy and Technology" Dec-2016 Vol. 8 Issue No.4 5049- 5054 